\title{
GCU
}

Glasgow Caledonian

University

University for the Common Good

\section{Validity and reliability of the activPAL3 for measuring posture and stepping in adults and young people}

Sellers, Ceri; Dall, Philippa; Grant, Margaret; Stansfield, Ben

Published in:

Gait and Posture

DOI:

10.1016/j.gaitpost.2015.10.020

Publication date:

2016

Document Version

Author accepted manuscript

Link to publication in ResearchOnline

Citation for published version (Harvard):

Sellers, C, Dall, P, Grant, M \& Stansfield, B 2016, 'Validity and reliability of the activPAL3 for measuring posture and stepping in adults and young people', Gait and Posture, vol. 43, pp. 42-47.

https://doi.org/10.1016/j.gaitpost.2015.10.020

\section{General rights}

Copyright and moral rights for the publications made accessible in the public portal are retained by the authors and/or other copyright owners and it is a condition of accessing publications that users recognise and abide by the legal requirements associated with these rights.

Take down policy

If you believe that this document breaches copyright please view our takedown policy at https://edshare.gcu.ac.uk/id/eprint/5179 for details of how to contact us. 
Title

Validity and reliability of the activPAL3 for measuring posture and stepping in adults and young people

\section{Authors}

Ceri Sellers ${ }^{\mathrm{a}}$, Philippa Dall ${ }^{\mathrm{a}}$, Margaret Grant ${ }^{\mathrm{a}}$, Ben Stansfield ${ }^{\mathrm{a}}$

${ }^{a}$ Institute for Applied Health Research, School of Health and Life Sciences, Glasgow Caledonian University, Cowcaddens Road, Glasgow, G4 OBA, UK.

E-mails: Ceri.Sellers@gcu.ac.uk, Philippa.Dall@gcu.ac.uk, M.Grant@gcu.ac.uk, ben.stansfield@gcu.ac.uk

Corresponding author: Ben Stansfield, Institute for Applied Health Research, School of Health and Life Sciences, Glasgow Caledonian University, Cowcaddens Road, Glasgow, G4 OBA, UK. ben.stansfield@gcu.ac.uk. Tel: 0044 (0) 1412731551.

Acknowledgements (funding)

The work completed here was funded as part of an EPSRC CASE PhD studentship award which was partly funded by PAL Technologies Ltd (Glasgow, UK), the manufacturer of the activPAL3 device. The authors would like to thank all participants for their time in taking part in this work. 


\section{Conflict of interest}

This work was funded jointly by the UK Engineering and Physical Sciences Research Council (EPSRC) (Grant reference EP/G501416/1) and PAL Technologies Ltd (Glasgow, UK) as part of a CASE PhD Studentship award. PAL Technologies Ltd is the manufacturer of the activPAL3, the device used in this study. Ceri Sellers was the funded PhD student and Ben Stansfield and Philippa Dall were named Investigators on the award. PAL Technologies Ltd had no involvement with the design, collection, analysis or interpretation of data, nor in the writing of the manuscript or the decision to submit the manuscript for publication. Ben Stansfield has previously received grant funding from PAL Technologies Ltd for an unrelated study. 
Highlights

The activPAL3 is valid for the detection of posture and purposeful stepping.

Posture detection was excellent for standardised activities.

Only purposeful steps during activities of daily living were detected by the monitor.

The activPAL3 demonstrates good to excellent $(\operatorname{ICC}(1,1))$ inter-device reliability. 


\section{Abstract}

Characterisation of free-living physical activity requires the use of validated and reliable monitors. This study reports an evaluation of the validity and reliability of the activPAL3 monitor for the detection of posture and stepping in both adults and young people. Twenty adults (median 27.6y; IQR22.6y) and 8 young people (12.0y; IQR4.1y) performed standardised activities and activities of daily living (ADL) incorporating sedentary, upright and stepping activity. Agreement, specificity and positive predictive value were calculated between activPAL3 outcomes and the gold-standard of video observation. Inter-device reliability was calculated between 4 monitors. Sedentary and upright times for standardised activities were within $\pm 5 \%$ of video observation as was step count (excluding jogging) for both adults and young people. Jogging step detection accuracy reduced with increasing cadence $>150$ steps/min. For ADLs, sensitivity to stepping was very low for adults (40.4\%) but higher for young people (76.1\%). Inter-device reliability was either good $(\operatorname{ICC}(1,1)>0.75)$ or excellent $(\operatorname{ICC}(1,1)>0.90)$ for all outcomes. An excellent level of detection of standardised postures was demonstrated by the activPAL3. Postures such as seat-perching, kneeling and crouching were misclassified when compared to video observation. The activPAL3 appeared to accurately detect 'purposeful' stepping during $A D L$, but detection of smaller stepping movements was poor. Small variations in outcomes between monitors indicated that differences in monitor placement or hardware may affect outcomes. In general, the detection of posture and purposeful stepping with the activPAL3 was excellent indicating that it is a suitable monitor for characterising free-living posture and purposeful stepping activity in healthy adults and young people.

Keywords

Validity; reliability; activPAL3 activity monitor; posture; steps; adults; children 


\section{INTRODUCTION}

Whilst laboratory-based observation of human movement can tell us what a person is capable of, it is necessary to make recordings within the person's free-living environment to develop an understanding of what they actually do. The measurement of this activity must be performed using instruments with demonstrated validity and reliability[1].

The activPAL activity monitor is a uni-axial activity monitor manufactured by PAL Technologies Limited, Glasgow, UK, with demonstrated validity and reliability for characterising posture and measuring stepping for adults[2-4], older adults[5], pre-school children[6], 9-10 year olds[7] and female adolescents[8,9].

The activPAL3 monitor, produced by the same company, contains a tri-axial accelerometer. It outputs a different range of raw acceleration and uses a higher sampling frequency and subsequently different hardware filtering compared to the earlier version of the activPAL. Therefore, demonstrated validity and reliability of the activPAL may apply to the activPAL3. Despite claims that the activPAL3 has been "widely validated"[10], only limited reports in adults are available. Berendsen et al (2014)[11] report 100\% validity for detecting posture type/walking time for 5 adults $(22.4 \pm 2.2 \mathrm{y})$ performing a highly controlled protocol eliminating transitions between activities. Stansfield et al (2014)[12] reported step counting accuracy during treadmill walking, demonstrating that steps are accurately counted above $0.5 \mathrm{~ms}^{-1}$. However, they reported only a limited range of stepping speeds also with transitions removed. Ryde et al (2012)[13] studied office workers for a short standardised protocol involving sitting and standing (3-60s duration) and $1 \mathrm{~h}$ of free-living office duties. They reported excellent agreement between direct observation and activPAL3 for sitting time and number of sit-to-stand transitions[13]. To enhance our understanding of the validity and reliability of the activPAL3, a protocol must be used which examines not only controlled standardised testing, but also incorporates elements of choice of movement pattern and activities, similar to a range of free-living conditions. There is also a need to expand the validation to 
children and adolescents, where differences in outcome may be expected due to smaller size and different movement pattern compared to adults.

The primary aims of this study were to determine the validity and inter-device reliability of the activPAL3 in measuring posture and stepping of adults and young people with unimpaired mobility.

To enhance the generalizability of outcomes to activity in free-living environments, protocols involving typical activities of daily living with partial free-choice were incorporated[3,4]. 


\section{METHODS}

\section{Participants}

Two convenience samples were recruited from university staff, students and their families:

- Adults: 20 participants aged $18-65$ years.

- Young People: 8 participants aged 6-17 years.

Participant numbers were recruited in line with previous study populations (5-30 per group) $[2,3,7,9,11-13]$. Participants had to be able to walk independently without mobility aids, carry out everyday tasks and leisure time activities independently and walk for $40 \mathrm{mins}$ within $80 \mathrm{mins}$. Ethical approval was gained from the institutional review board. Age-appropriate information sheets were provided to participants and parents of the young people. All participants gave informed consent/assent.

\section{General procedure}

Participants were asked to wear the activPAL3 and carry out activities while being videoed. Posture and stepping measures recorded by the activPAL3 were compared against the video observation criterion measure.

\section{Physical activity monitor}

Posture and stepping were measured using the activPAL3, worn on the front of the thigh (PAL Technologies Ltd, Glasgow, UK). The monitor uses proprietary analysis algorithms to determine posture (sedentary time, upright time) and stepping (stepping time and steps). Each participant was fitted with four activPAL3 monitors randomly selected from a pool of 10. ActivPAL3 monitors were affixed to the skin with hydrogel pads as close as possible to the manufacturer's recommended 
position of a third of the way down the anterior thigh[14], three on the right leg (two piggybacked one on top of the other) and one on the left (supplementary figure S1). Placement was made by eye as no anatomical frame of reference for placement was provided by the manufacturer.

\section{Protocol}

Testing lasted approximately $1 \mathrm{~h} 20 \mathrm{mins}$ (Table 1 ) with task order randomised within two sections. Test activity timings were recorded using a digital watch which had been synchronised with the laptop used to program the activPAL3 monitors.

- Standardised Activities - Fourteen activities (8 inside, 6 outside) (Table 1) were performed. Participants stood for $15 \mathrm{~s}$ before and after performing each activity to provide a break in the activPAL3 record. Outdoor activities were performed on paved surfaces (including kerbs and slight slopes) using a set route.

- Activities of Daily Living (ADL) - Intended to represent activities that participants might carry out in their daily lives. A total of 18 tasks (Table 1) were identified for adults[3] and 14 for young people. Tasks were assigned to lists of 6 activities utilising a range of postures. Each participant completed one randomly-assigned task list of 6 activities. Participants sat for $15 \mathrm{~s}$ between activities.

\section{Analysis}

Video recordings were analysed by a single researcher classifying time as stepping, standing or sedentary (sitting/lying) and identified steps taken. Activities for one participant were independently categorised by an additional researcher to evaluate the integrity of definitions. Standing was defined as any time when participants were on two feet supporting their full body weight, sitting when weight was supported. Steps were defined as any action where the foot left, 
then reconnected with the floor. This included both 'purposeful', directed stepping and small incidental stepping. ActivPAL3 data was processed using activPAL software version 7.1.18 (minimum sitting and standing period $2 \mathrm{~s}[15])$. Data from video observation and activPAL3 were aggregated into totals for standardised activities and ADL sections separately and the following outcomes were measured:

- Duration: Total time spent sedentary (sitting/lying), upright, standing and stepping

- Step Count: Total number of steps taken.

Stepping activity was examined using all steps taken (including jogging), all steps without jogging and for jogging alone. An additional second-by-second analysis compared observed and activPAL3 recorded posture.

\section{Validity}

The activPAL3 closest to the manufacturer's recommended position of $1 / 3$ of the way down the thigh was used for the validation analysis. Outcome measures were analysed using modified Bland and Altman plots[16] with:

$$
\text { y axis: } \frac{100 \times\{\text { total Ob. seconds (or steps) }- \text { total activPAL3 seconds (or steps) }\}}{\text { total Ob. seconds (or steps) }}
$$

$\mathrm{x}$ axis: mean of total Ob. seconds (or steps) and total activPAL3 seconds (or steps)

Where Ob. = observed by video assessment and activPAL3 = activity monitor outcomes. Upper and lower limits of agreement were calculated as $\pm 1.96 \mathrm{SD}$. Apriori limits for acceptability of difference between activPAL3 and video were set to $\pm 5 \%[17]$.

The difference between the posture recorded by video and activPAL3 was also compared for each second to assess whether patterns of activity were being accurately recorded. Percentage agreement, sensitivity and positive predictive value (PPV) were calculated[18]: 


$$
\% \text { agreement }=\frac{\text { Number of seconds where activPAL3 posture }=\text { observed posture }}{\text { Number of seconds }} \times 100
$$

Sensitivity $=\frac{\text { No. of seconds where activPAL3 posture }=\mathrm{A} \text { and observed posture }=\mathrm{A}}{\text { Number of seconds where observed posture }=\mathrm{A}} \times 100$

$$
\mathrm{PPV}=\frac{\text { Number of seconds where activPAL3 posture }=\mathrm{A} \text { and observed posture }=\mathrm{A}}{\text { Number of seconds where activPAL3 }=\mathrm{A}} \times 100
$$

Using sitting as an example, sensitivity indicates the percentage of true sitting that the monitor is successfully reporting, while PPV gives the percentage of sitting reported by the monitor that is correct. Agreement provides the percentage of time the video and monitor agree.

\section{Reliability}

Data from all four monitors was used to calculate inter-monitor reliability. Inter-device agreement was calculated using the ICC $(1,1)$ form of the Intraclass Correlation Coefficient[19]:

$$
\operatorname{ICC}(1,1)=\frac{B M S-W M S}{B M S+(k-1) W M S}
$$

Where: $B M S=$ between targets mean square, $W M S=$ within-groups (error) mean square, $K=$ number of monitors. ICC values of at least 0.75 were rated good and $>0.90$ excellent[20]. 


\section{RESULTS}

Twenty adults (9M,11F) (median age 27.6y (IQR 22.6), mean height $172.4 \pm 9.0 \mathrm{~cm}$, weight $73.3 \pm 13.0 \mathrm{~kg}, \mathrm{BMI} 24.6 \pm 3.2 \mathrm{~kg} / \mathrm{m}^{2}$ ) and 8 young people $(2 \mathrm{M} / 6 \mathrm{~F}$ ) (mean age $12.0 \pm 4.1 \mathrm{y}$, height $152.4 \pm 25.0 \mathrm{~cm}$, weight $42.3 \pm 16.4 \mathrm{~kg}$ ) took part. All young people were normal weight. Self-selected walking speeds were: Adults slow 0.98-1.61, normal 1.30-1.88, fast 1.61-2.24, treadmill jogging 1.43.0, outside jogging 2.2-4.1 $\mathrm{ms}^{-1}$; young people slow 0.92-1.53, normal 1.08-1.72, fast 1.53-2.06, treadmill jogging 2.1-2.9, outside jogging 2.1-3.3ms $\mathrm{m}^{-1}$.

A comparison of the categorisation of activity between researchers for one participant demonstrated no differences in posture or step detection for standardised activity and only minor differences for step detection in ADL for very small stepping movements.

Duration of standardised activities for adults was $25.5 \pm 1.2 \mathrm{mins}$ (stepping $19.2 \pm 1.2 \mathrm{mins}$ ) (supplementary table S1) and young people $24.1 \pm 3.1 \mathrm{mins}$ (stepping $17.8 \pm 2.5 \mathrm{mins}$ ). The ADL test duration for both adults (11.1 $\pm 1.5 \mathrm{mins})$ and young people (10.8 $\pm 3.0 \mathrm{mins})$ was lower with time spent more evenly between sedentary, standing and stepping activity.

Data was successfully collected for the majority of participants; one of four monitors worn by one adult failed to record activity; one young person did not carry out the treadmill jogging activity as her footwear was deemed unsafe. The two youngest participants carried out 'external' activities along a corridor due to adverse weather, reducing stepping distances to $55 \mathrm{~m}$ walking and $23 \mathrm{~m}$ jogging.

\section{Validity}

In adults, for both standardised and ADL activities, activPAL3 and video durations were very similar for sedentary and upright (supplementary table S1). A small proportion of the standardised activity stepping was misclassified as standing. Consequently step count was slightly underestimated for all 
standardised activity (3.45\% for adults). This difference was far greater for ADL testing, with a much higher undercount of steps. For young people standardised activity outcomes were broadly similar to adults. Also for some of the young people during ADLs some sitting was misclassified as standing and more stepping time was detected by the activPAL3 than by video observation.

Modified Bland and Altman plots indicated that a number of outcomes did meet the apriori limit of $\pm 5 \%$ difference (Table 2); these included sedentary and upright times for standardised activity (adult and young people) and ADL (adults only), stepping duration for standardised activity and step counts excluding jogging (adults and young people). No obvious bias was apparent in the plots for these measures (supplementary figure S2). There was poor agreement for standardised activity step count (jogging only), ADL step count and ADL stepping duration (adults only).

There was a high level of second by second agreement between activPAL3 and video observation for standardised activities for both adults (min 97.9\%) and young people ( $\min 95.0 \%$ ). Results were lower for ADL activities, particularly for young people ( $\min 75.4 \%$ ). Categorising activity into 3 states (stepping, standing and sedentary), demonstrated a high level of sensitivity for standardised activities for adults ( $\min 97.2 \%)$ and young people ( $\min 94.0 \%)$. However, standardised activity PPV was lower for both adults ( $\min 84.3 \%$ ) and young people $(\min 62.8 \%$ ). For ADLs, sensitivity to stepping was very low for adults (40.4\%), with low PPV for standing $(75.1 \%)$ and stepping $(70.6 \%)$. For young people both sensitivity and PPV were low for ADL standing and stepping (57.8-76.1\%).

\section{Reliability}

Outputs of four activPAL3 monitors were used to calculate reliability. One adult participant was excluded from this analysis as one of the monitors failed. The monitors mounted in the two lower right leg positions misclassified some sitting activity as standing in the standardised testing for three adults and three young people. The monitors placed higher up the thigh correctly identified sitting, 
while those in the lower leg positions categorised sitting activity as standing. Excluding these specific results, reliability was excellent $(\operatorname{ICC}(1,1)>0.90)$ for all outcome measures for adults except standardised activity standing duration, ADL stepping duration and ADL step count, which were good $(\operatorname{ICC}(1,1)>0.75)$. For young people, reliability was excellent for all outcomes except for ADL upright duration, $A D L$ standing duration and $A D L$ step count, which were good. 


\section{DISCUSSION}

It is necessary to be cautious in assuming that new or 'upgraded' monitors, with different hardware and software, produce the same outcomes as older monitors. Therefore, new physical activity monitors must be assessed for validity and reliability, ideally under conditions representative of their intended use. To achieve this aim for the activPAL3, the current study implemented both a standardised, controlled protocol and an ADL, relatively self-selected, protocol. The assessment of the activPAL3 was also extended to children, which has not been reported before. Sedentary, upright and stepping time and steps (without jogging) were all detected for standardised activities with LOA $< \pm 5 \%$. However, for jogging activities for both adults and young people, steps were undercounted to an increasing degree as cadence increased. For the ADL activities there were considerably lower levels of agreement, especially for step count. When placed at the manufacturers recommended location, inter-device reliability was in general excellent. However, this reduced for aspects of $A D L$, especially step detection.

\section{Strengths and Limitations}

The number of participants, although in line with previous research in this area, was relatively small. It is possible that this might limit the applicability of the outcomes to the wider population of adults and children, especially to clinical and/or obese populations. However, these results provide a substantial improvement on current evidence. The inclusion of young people in comparison to adults highlights important differences in monitor performance. Aspects of young people's movements which provide disagreement between video observation and monitor outcomes (e.g. seat perching) are highlighted. It is possible that an extended sample may reveal more specific activities where disagreement occurs. However, the high levels of agreement (with narrow confidence intervals) for standardised activities provide confidence that the results would be representative of the wider population. Within the ADL tasks self-selected postures and movements were allowed. Also periods 
of acceleration and deceleration were included in the analysis. Therefore, the analysis goes some way towards providing an assessment of monitor performance under free-living conditions. However, the layout of the laboratory and the distances walked $(3-6 \mathrm{~m})$ during ADL activities cannot represent all environments, potentially limiting generalizability of the results.

\section{Sedentary/upright classification}

The activPAL3 demonstrated excellent agreement (Table 2) with video observation for the separation sedentary and upright time in all cases except for young people ADL activities. During standardised activities the outcomes were in agreement with previous studies. For example Berendsen et al[11] reported $100 \%$ correct detection of sitting and standing using a highly prescribed protocol. Ryde et al[13] report lower levels of agreement $(0.49 \mathrm{mins}$ under detection of sitting in 4.52mins). However, their protocol used multiple short sitting events.

In the current study, for ADL, incorrect separation of standing and sitting time for young people was associated with perching on the edge of chairs by smaller children (previously reported for activPAL in children with cerebral palsy[21] and pre-school children[6]) and adoption of crouching or kneeling postures during some activities (supplementary figure S3). As the activPAL3 is thigh-mounted the thigh angle is critical in determining posture. The angulation occurring was sufficient, in some cases, to cause misclassification of sitting with standing. It is not possible to say how perching, kneeling and crouching might impact upon free-living data interpretation as the extent to which these postures are adopted has not been documented.

\section{Standing/stepping classification}

Overall, for standardised activities, step detection by the activPAL3 was similar to video interpretation. However, only when jogging activities were removed were limits of agreement 
within $\pm 5 \%$. A slight stepping undercount remained, which could be explained by the inclusion of the acceleration and deceleration phases of stepping activities within this protocol. Also, as the software reports strides (steps=2xstrides), initiating and terminating steps may not have been detected leading to undercounting. For jogging, as cadence of stepping increased, the proportion of steps detected reduced suggesting that the monitor undercounts faster jogging steps (>150steps/min) (Figure 1). For only non-jogging steps, during standardised activities, step (and associated stepping time) detection was excellent indicating that steps taken when walking at speeds from $0.92-2.24 \mathrm{~ms}^{-1}$ were correctly detected.

For ADL activities, steps were under-detected to a large degree. However, this was not consistently associated with a lower stepping time. This indicates that the activPAL3 was classifying stepping time, but not detecting steps, leading to the classification of artificially long, low cadence steps. The results of the current study, i.e. poor slow stepping detection $\left(<0.5 \mathrm{~ms}^{-1}\right)$, but good self-selected speed step detection $\left(>0.9 \mathrm{~ms}^{-1}\right)$, are in agreement with previous reports[12]. This suggests that steps not detected were those less 'purposeful' taken during the ADLs. To investigate the significance of step 'size' further categorisation of steps into 'purposeful' (involving progression), medium (some progression, small steps) and small (limited progression, small steps) was made subjectively for several of the ADL activities. Graphical examination of agreement of activPAL3

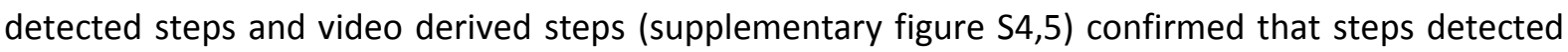
by the activPAL3 were in general 'purposeful'. Difficulties with creating a clear, unambiguous definition of stepping type made further investigation of the exact nature of detected steps difficult.

\section{Inter-device reliability}

It was not possible to place 4 monitors used to examine inter-device reliability in exactly the same location. This difference in positioning meant that the monitors were using slightly different accelerometer signals to derive classifications of posture and stepping. Critically, it appears that 
monitors attached lower down the thigh were detecting different postures depending on the shape/curvature of the thigh. As the intention was to assess inter-device reliability rather than the effect of positional changes the data sets were reduced to remove those cases where the lower monitors gave different outcomes. With these cases removed all outcomes demonstrated either good or excellent reliability. For the key outcomes of sedentary time, upright time and step count ICC $(1,1)$ values of 0.94 and above were recorded for standardised activities (Table 4). Differences for the ADL activities arose for the detection of stepping (ICC $(1,1)$ adults $95 \% \mathrm{Cl} 0.63-0.90$, young people 0.73-0.97), indicating that different monitors were detecting different numbers of steps, but still with good levels of reliability. These differences may have been due to the slight differences in monitor placement on the thigh, or to device hardware differences (e.g. accelerometer alignment).

\section{Summary}

The activPAL3 determined sitting/lying and upright postures excellently for standardised activities. However, for ADL activities some misclassification occurred due to seat-perching, kneeling and crouching. Step detection for standardised activities was good, but for jogging, as cadence increased, the proportion of steps detected decreased. Also for ADL activities the activPAL3 appeared to only capture 'purposeful' steps. Inter-device reliability was generally excellent for standardised activities, but slightly lower for ADL activities indicating that differences in device placement/hardware configuration may affect outcomes. In this sample the detection of posture and purposeful stepping with the activPAL3 was excellent indicating that it is a suitable monitor for characterising these aspects of free-living activity in healthy adults and children. 


\section{REFERENCES}

[1] Campbell MJ, Machin D. Medical statistics: A common sense approach. 3rd ed. London: John Wiley \& Sons; 1999.

[2] Ryan CG, Grant PM, Tigbe WW, Granat MH. The validity and reliability of a novel activity monitor as a measure of walking. Br J Sports Med 2006; 40: 779-84.

[3] Grant PM, Ryan CG, Tigbe WW, Granat, MH. The validation of a novel activity monitor in the measurement of posture and motion during everyday activities. Br J Sports Med 2006; 40: 992-7.

[4] Ryan CG, Grant PM, Gray H, Newton M, Granat MH. Measuring postural physical activity in people with chronic low back pain. J Back Musculoskelet Rehabil 2008; 21(1): 43-50.

[5] Grant PM, Dall PM, Mitchell SL, Granat MH. Activity-monitor accuracy in measuring step number and cadence in community-dwelling older adults. J Aging Phys Act 2008; 16(2): 201-14.

[6] Davies G, Reilly J, McGowan A, Dall P, Granat M, Paton J. Validity, practical utility, and reliability of the activPAL in preschool children. Med Sci Sports Exerc 2012; 44(4): 761-8.

[7] Aminian S, Hinckson EA. Examining the validity of the ActivPAL monitor in measuring posture and ambulatory movement in children. Int J Behav Nutr Phys Act 2012; 9: 119.

[8] Harrington D. Objective assessment and modification of physical activity and health indices in adolescent females [dissertation]. Limerick, Ireland: University of Limerick; 2010.

[9] Dowd KP, Harrington DM, Donnelly AE. Criterion and concurrent validity of the activPALTM professional physical activity monitor in adolescent females. PLoS One 2012; 7(10): e47633.

[10] Lowe SA, ÓLaighin G. Monitoring human health behaviour in one's living environment: A technological review. Med Eng Phys 2014; 36: 147-68.

[11] Berendsen BAJ, Hendriks MRC, Meijer K, Plasqui G, Schaper NC, Savelberg HHCM. Which activity monitor to use? Validity, reproducibility and user friendliness of three activity monitors. BMC Public Health 2014; 14: 749. 
[12] Stansfield B, Hajarnis M, Sudarshan R. Characteristics of very slow stepping in healthy adults and validity of the activPAL3 ${ }^{\text {TM }}$ activity monitor in detecting these. Med Eng Phys 2015; 37: 42-7.

[13] Ryde GC, Gilson ND, Suppini A, Brown WJ. Validation of a novel, objective measure of occupational sitting. J Occup Health 2012; 54: 383-6.

[14] PAL Technologies Ltd. ACTIVPAL 3тм operating guide. Glasgow: PAL Technologies Ltd; 2010.

[15] Alghaeed Z, Reilly JJ, Chastin SFM, Martin A, Davies G, Paton JY. The influence of minimum sitting period of the ActivPAL ${ }^{\mathrm{TM}}$ on the measurement of breaks in sitting in young children. PLoS One 2013; 8(8): e71854.

[16] Bland JM, Altman DG. Statistical methods for assessing agreement between two methods of clinical measurement. Lancet 1986, i: 307-10.

[17] Dewitte K, Fierens C, StöckI D, Thienpont LM. Application of the Bland-Altman plot for interpretation of method-comparison studies: A critical investigation of its practice. Clin Chem 2002; 48: $799-801$.

[18] Bussmann HBJ, Reuvekamp PJ, Veltink PH, Martens WLJ, Sta HJ. Validity and reliability of measurements obtained with an "activity monitor" in people with and without a transtibial amputation. Phys Ther 1998; 78(9): 989-98.

[19] Shrout PE, Fleiss JL. Intraclass correlations: Uses in assessing rater reliability. Psychol Bull 1979; 86(2): 420-8.

[20] Portney LG, Watkins MP. Foundations of clinical research. Applications to practice, East Norwalk CT: Appleton \& Lange; 1993.

[21] Tang KT, Richardson AM, Maxwell D, Spence WD, Stansfield BW. Evaluation of an activity monitor for the objective measurement of free-living physical activity in children with cerebral palsy. Arch Phys Med Rehabil 201 


\section{List of Figures}

Figure 1: Adults and young people (YP) jogging at self-selected speed - step count comparison between activPAL3 and video 


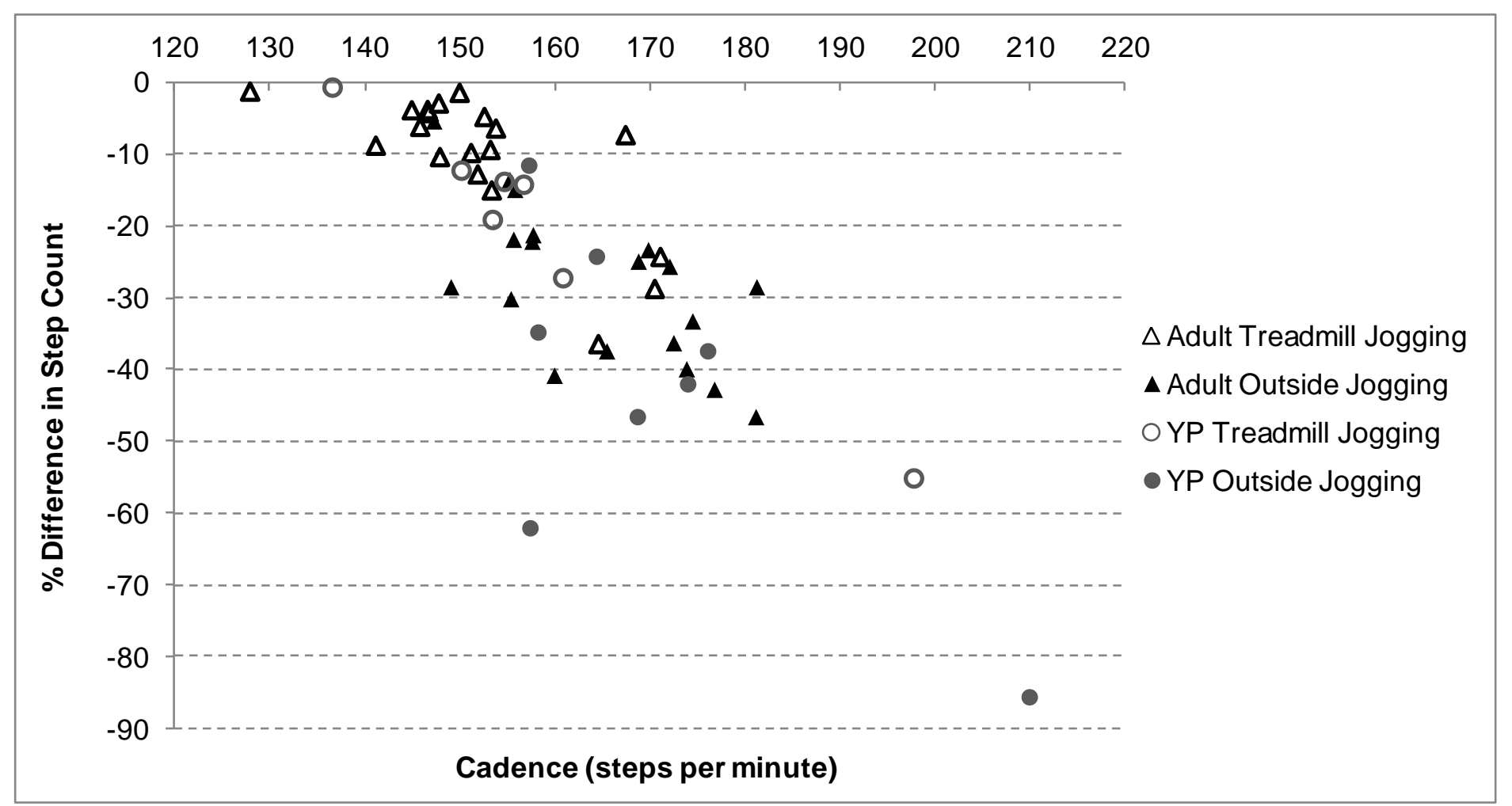

Figure 1: Adults and young people (YP) jogging at self-selected speed - step count comparison between activPAL3 and video 
Table 1: Standardised and daily living (ADL) activities for both adults and children.

\begin{tabular}{|c|c|c|}
\hline Standardised Activities & Activities of Daily living (ADL) (indc & rs) \\
\hline $\begin{array}{l}\text { Indoors - } 8 \text { tasks, } 2 \text { mins each } \\
\text { Sit } \\
\text { Lie } \\
\text { Stand } \\
\text { Walk on treadmill at } 4 \text { different speeds: } \\
\text { Adults, young people >= } 11 \text { years } 0.90,1.12 \text {, } \\
1.33,1.57 \mathrm{~ms}^{-1} \\
\text { Young people < } 11 \text { years } 0.67,0.90,0.12,1.33 \\
\text { ms }^{-1} \\
\text { Jog on treadmill (self-selected speed) } \\
\text { Outdoors - } 6 \text { tasks } \\
200 \mathrm{~m} \text { walk normal speed } \\
200 \mathrm{~m} \text { walk fast-speed } \\
200 \mathrm{~m} \text { walk slow-speed } \\
50 \mathrm{~m} \text { jogging (self-selected speed) } \\
\text { Descend } 15 \text { steps } \\
\text { Ascend } 15 \text { steps }\end{array}$ & $\begin{array}{l}\text { Adults - } 6 \text { tasks (2-5mins) [3] } \\
\text { Hang washing out to dry } \\
\text { Take clothes off a clothes rack } \\
\text { Put on duvet cover and pillowcases } \\
\text { Putting the rubbish out } \\
\text { Wash and dry hands } \\
\text { Change bulb in table lamp } \\
\text { Place lampshade on table lamp } \\
\text { Make and drink hot or cold drink } \\
\text { Remove clothes from basket and iron } \\
\text { Word-process document using PC } \\
\text { Watch a DVD } \\
\text { Read newspaper } \\
\text { Clean a framed picture } \\
\text { Wash and dry dishes } \\
\text { Vacuuming } \\
\text { Write letter/list } \\
\text { Make a mobile phone call }\end{array}$ & $\begin{array}{l}\text { Young People - } 6 \text { tasks (1-4mins) } \\
\text { Make and drink cold drink } \\
\text { Taking a coat/cardigan off a coat hook and } \\
\text { putting on. } \\
\text { Keepy-uppy (football/balloon) } \\
\text { Kicking football at goal } \\
\text { Skipping } \\
\text { Indoor basket ball } \\
\text { Throwing bean bags at a target } \\
\text { Using a computer } \\
\text { Watch a DVD } \\
\text { Reading } \\
\text { Drawing } \\
\text { Card game } \\
\text { Basketball } \\
\text { Hula hoop }\end{array}$ \\
\hline
\end{tabular}


TABLE 2 Bland and Altman Percentage Mean Differences: Video Observation and activPAL3

\begin{tabular}{|c|c|c|c|c|}
\hline \multirow{3}{*}{ Measure } & \multicolumn{4}{|c|}{ Percentage mean difference (LLOA, ULOA) (\%) } \\
\hline & \multicolumn{2}{|c|}{ Standardised Activities } & \multicolumn{2}{|c|}{ ADL Activities } \\
\hline & Adults & Young people & Adults & Young people \\
\hline \multicolumn{5}{|l|}{ Duration } \\
\hline Sedentary & $-0.07(-0.28,0.15)^{* *}$ & $-0.28(-1.23,0.66)^{* *}$ & $0.53(-0.96,2.02)^{* *}$ & $-2.84(-124.98,130.67)$ \\
\hline Upright & $0.27(-0.24,0.77)^{* *}$ & $0.28(-0.17,0.73)^{* *}$ & $-0.19(-2.08,1.70)^{* *}$ & $9.03(-39.17,55.23)$ \\
\hline Standing & $15.16(5.31,25.01)$ & $17.6(9.97,25.22)$ & $19.67(-1.21,40.55)$ & $5.55(-79.95,91.05)$ \\
\hline Stepping & $-1.42(-2.68,-0.17)^{* *}$ & $-1.76(-3.25,-0.28)^{* *}$ & $-54.91(-95.18,-14.63)^{\#}$ & $14.09(-15.68,43.85)$ \\
\hline \multicolumn{5}{|l|}{ Step Count } \\
\hline All activities & $-3.45(-7.31,0.42)$ & $-5.68(-14.75,3.38)$ & $-86.23(-117.14,-55.31)^{\#}$ & $-36.51(-14.40,-58.62)^{\#}$ \\
\hline All activities excluding jogging & $-1.33(-2.74,0.07)^{* *}$ & $-1.71(-3.30,-0.11)^{* *}$ & & \\
\hline Jogging activities only & $-13.66(-35.20,7.88)^{\#}$ & $-29.72(-76.68,17.25)^{\#}$ & & \\
\hline
\end{tabular}

"poor visual agreement. ${ }^{* *}$ met the criteria of $\pm 5 \%$ limits of agreement. LLOA=lower limits of agreement, ULOA=upper limits of agreement. ADL=activities of daily living. 
Table 3: Second-by-second posture agreement, sensitivity and PPV: Video observation and activPAL3

\begin{tabular}{|c|c|c|c|c|c|c|c|c|c|c|c|c|}
\hline \multirow[b]{3}{*}{ Measure } & \multicolumn{6}{|c|}{ Standardised Activities } & \multicolumn{6}{|c|}{ ADL Activities } \\
\hline & \multicolumn{3}{|c|}{ Adults } & \multicolumn{3}{|c|}{ Young people } & \multicolumn{3}{|c|}{ Adults } & \multicolumn{3}{|c|}{ Young people } \\
\hline & 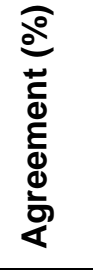 & 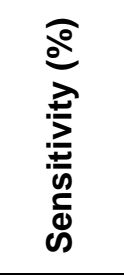 & $\frac{\widehat{o}}{\stackrel{0}{a}}$ & 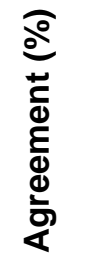 & 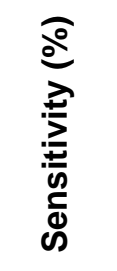 & $\frac{\grave{o}}{\stackrel{0}{a}}$ & 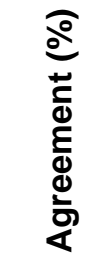 & 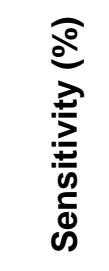 & $\frac{\widehat{o}}{\stackrel{0}{a}}$ & 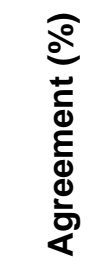 & 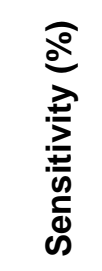 & $\frac{\widehat{o}}{\stackrel{0}{a}}$ \\
\hline Upright, Sedentary & 99.8 & & & 99.5 & & & 97.0 & & & 86.8 & & \\
\hline Sedentary & & 99.2 & 100.0 & & 97.0 & 100.0 & & 98.3 & 96.9 & & 84.2 & 89.6 \\
\hline Upright & & 100.0 & 99.8 & & 100.0 & 99.3 & & 95.1 & 97.0 & & 91.1 & 85.2 \\
\hline $\begin{array}{l}\text { Stepping, Standing, } \\
\text { Sedentary }\end{array}$ & 97.9 & & & 95.0 & & & 87.6 & & & 75.4 & & \\
\hline Standing & & 99.9 & 84.3 & & 100.0 & 62.8 & & 88.5 & 75.1 & & 57.8 & 61.0 \\
\hline Stepping & & 97.2 & 100.0 & & 94.0 & 100.0 & & 40.4 & 70.6 & & 76.1 & 64.8 \\
\hline
\end{tabular}


Table 4: Intraclass Correlation Coefficient $(\operatorname{ICC}(1,1))$ for standardised activities and ADL for adults and young people.

\begin{tabular}{|l|c|c|c|c|}
\hline \multirow{2}{*}{$\begin{array}{l}\text { Outcome Measure } \\
\text { ICC(1,1) (95\% Cl) }\end{array}$} & Adults & Young people & Adults & Young people \\
\hline Duration & & & & \\
Sitting/lying & $0.99(0.99,>0.99)^{1}$ & $0.98(0.90,>0.99)^{2}$ & $0.99(0.98,>0.99)$ & $0.91(0.77,0.98)$ \\
Upright & $>0.99(0.99,>0.99)^{1}$ & $0.94(0.84,0.99)^{2}$ & $0.99(0.98,0.99)$ & $0.89(0.73,0.97)$ \\
Standing & $0.88(0.77,0.95)^{1}$ & $0.98(0.90,>0.99)^{2}$ & $0.97(0.94,0.99)$ & $0.86(0.67,0.97)$ \\
Stepping & $>0.99(0.99,>0.99)$ & $>0.99(>0.99,1.00)$ & $0.82(0.68,0.92)$ & $0.90(0.74,0.98)$ \\
\hline Step Count & & & \\
All activities & $0.99(0.98,>0.99)$ & $>0.99(>0.99,>0.99)$ & $0.78(0.63,0.90)$ & $0.89(0.73,0.97)$ \\
\hline \multicolumn{4}{|c|}{${ }^{1}$ Based on data for 16 participants. ${ }^{2}$ Based on data from 5 participants. } \\
& \multicolumn{4}{|c|}{$>0.75$ Good $>0.90$ Excellent } \\
\hline
\end{tabular}




\section{Supplementary material}

\section{List of supplementary tables}

Table S1: Group Averages for Duration and Step Count: Video Observation and activPAL3

\section{List of supplementary figures}

Figure S1: Location of the activPAL3 monitors on the right and left thighs. Note that on the right leg the lower two monitors were stacked.

Figure S2:

A) Adults - Bland \& Altman Plots (average of monitor and video outcomes against \% difference between outcomes).

B) Young People - Bland \& Altman Plots (average of monitor and video outcomes against \% difference between outcomes).

Figure S3: Different classification examples (A adult, B-E young people). A) Video observation=sitting, activPAL3=standing; B-D) video=standing, activPAL3=sitting; E) video=sitting, activPAL3=standing.

Figure S4: Adult observed steps versus recorded strides for a participant (P6) for the empty rubbish bin ADL activity

Figure S5: Young people observed steps versus recorded strides for a participant (C5) for the coat On/Off ADL activity 
Table S1: Group Averages for Duration and Step Count: Video Observation and activPAL3

\begin{tabular}{|c|c|c|c|c|c|c|c|c|}
\hline \multirow{3}{*}{$\begin{array}{l}\text { Measure } \\
\text { All figures are Mean } \pm \text { SD }\end{array}$} & \multicolumn{4}{|c|}{ Standardised Activities } & \multicolumn{4}{|c|}{ ADL Activities } \\
\hline & \multicolumn{2}{|c|}{ Adults } & \multicolumn{2}{|c|}{ Young people } & \multicolumn{2}{|c|}{ Adults } & \multicolumn{2}{|c|}{ Young people } \\
\hline & Video & activPAL3 & Video & activPAL3 & Video & activPAL3 & Video & activPAL3 \\
\hline \multicolumn{9}{|l|}{ Duration (mins) } \\
\hline Sedentary & $4.3 \pm 0.1$ & $4.3 \pm 0.1$ & $4.5 \pm 0.4$ & $4.5 \pm 0.4$ & $4.7 \pm 1.1$ & $4.8 \pm 1.1$ & $4.4 \pm 1.8$ & $3.8 \pm 1.7$ \\
\hline Upright & $21.2 \pm 1.1$ & $21.3 \pm 1.1$ & $19.6 \pm 2.7$ & $19.6 \pm 2.7$ & $6.3 \pm 1.7$ & $6.2 \pm 1.7$ & $6.4 \pm 1.2$ & $7.0 \pm 1.4$ \\
\hline Standing & $2.0 \pm 0.2$ & $2.3+0.2$ & $1.9 \pm 0.3$ & $2.2 \pm 0.4$ & $4.3 \pm 1.5$ & $5.1 \pm 1.6$ & $3.5 \pm 1.1$ & $3.8 \pm 1.3$ \\
\hline Stepping & $19.2 \pm 1.2$ & $18.9 \pm 1.1$ & $17.7 \pm 2.5$ & $17.4 \pm 2.5$ & $2.0 \pm 0.6$ & $1.1 \pm 0.4$ & $2.9 \pm 0.8$ & $3.2 \pm 0.5$ \\
\hline \multicolumn{9}{|l|}{ Step Count (steps) } \\
\hline All activities & $2,233 \pm 181$ & $2,156 \pm 160$ & $2,128 \pm 292$ & $2,012 \pm 287$ & $207 \pm 53$ & $81 \pm 18$ & $337 \pm 91$ & $230 \pm 50$ \\
\hline All activities excluding jogging & $1,820 \pm 113$ & $1,793+106$ & $1,746 \pm 181$ & $1,717 \pm 183$ & & & & \\
\hline Jogging activities only & $438 \pm 47$ & $383 \pm 48$ & $382 \pm 146$ & $295 \pm 131$ & & & & \\
\hline
\end{tabular}




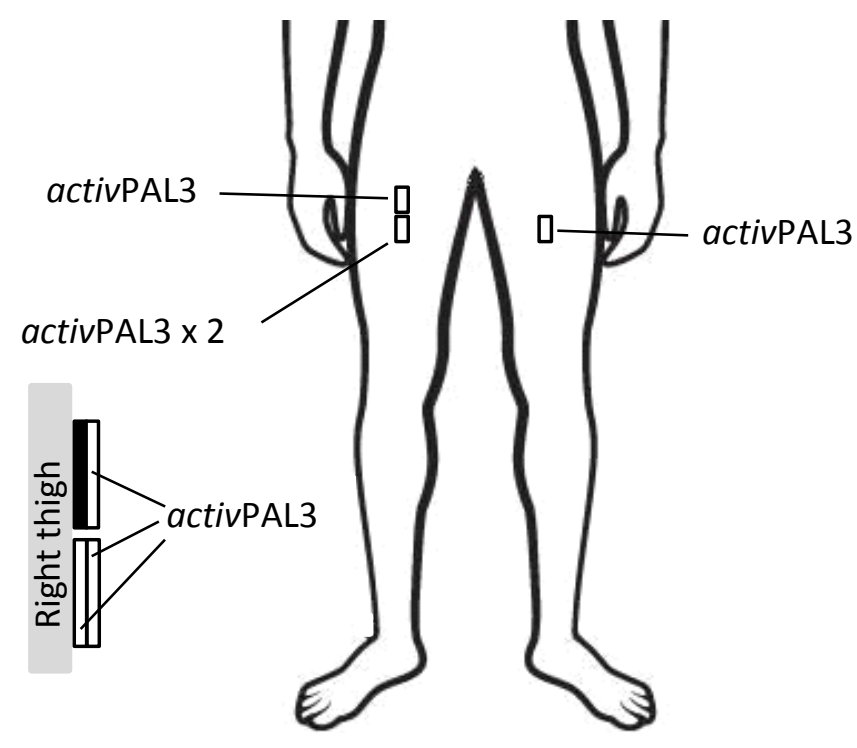

Figure S1: Location of the activPAL3 monitors on the right and left thighs. Note that on the right leg the lower two monitors were stacked. 
Figure S2 A) Adults - Bland \& Altman Plots (average of monitor and video outcomes against \% difference between outcomes) Sitting duration

\section{Upright duration}

Standardised activities (STD)

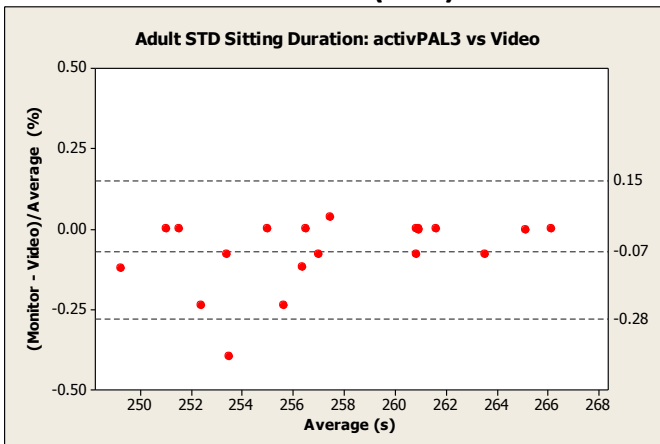

\section{Activities of daily living (ADL)}

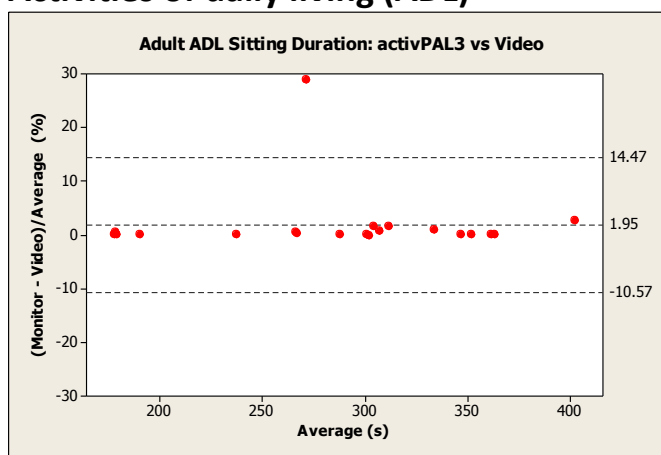

\section{Step count}

Standardised activities (STD)

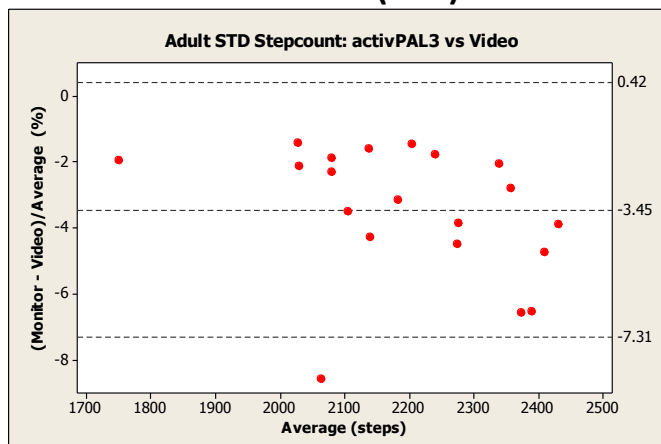

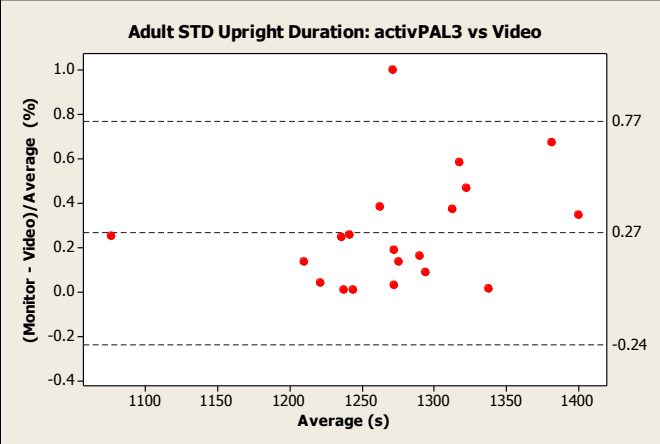
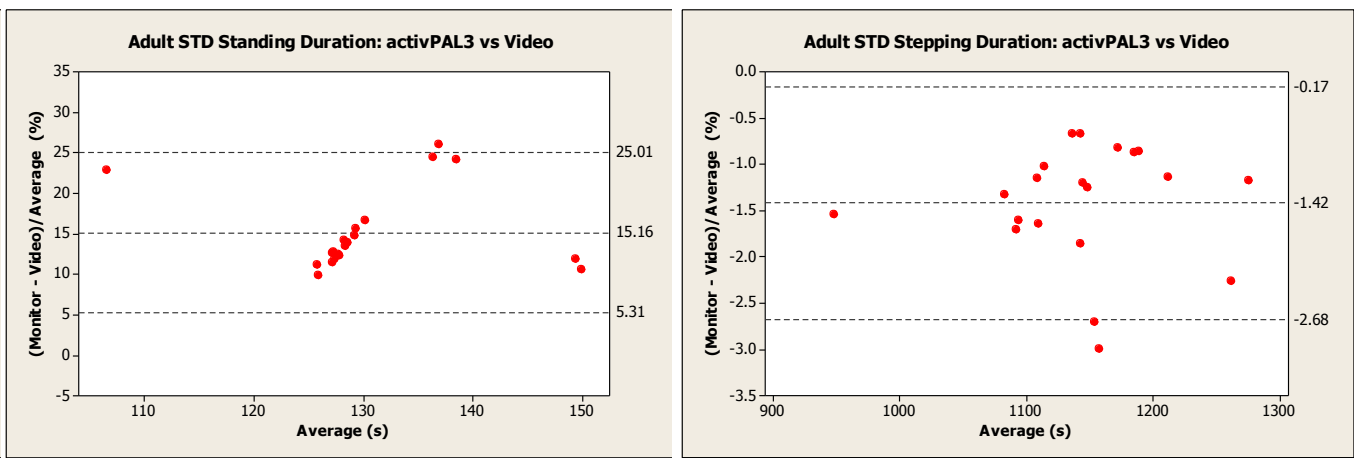

Stepping duration

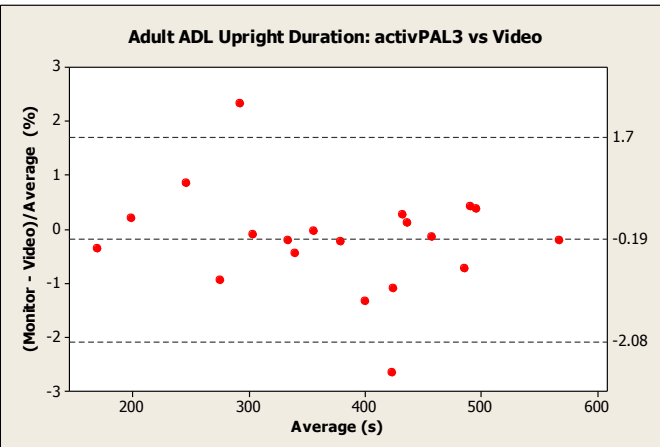

Step count excluding jogging

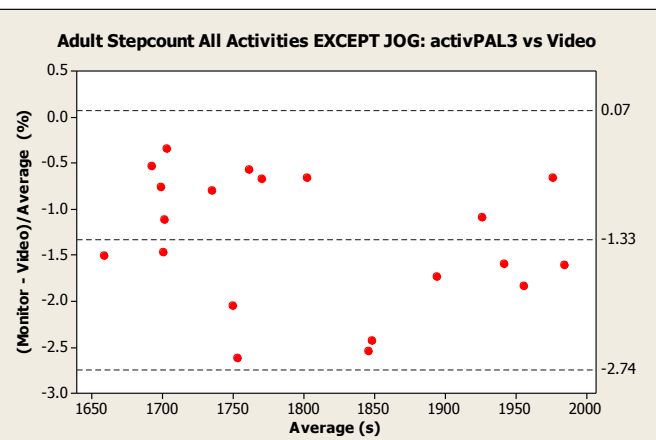

Adult ADL Standing Duration: activPAL3 vs Video

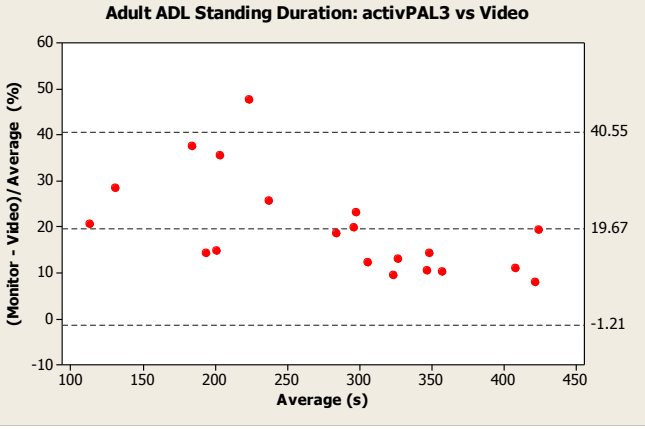

Step count jogging only

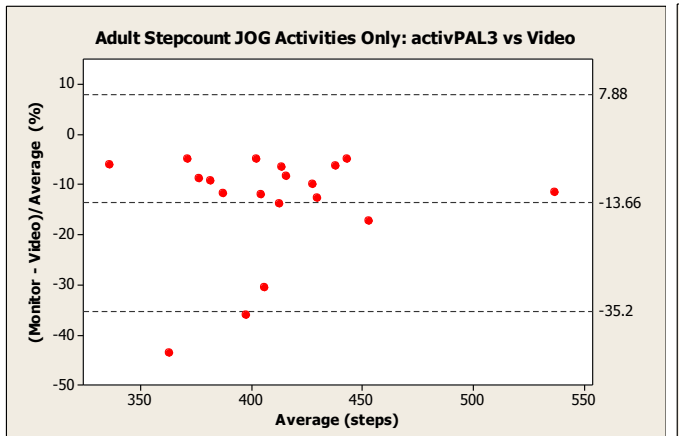

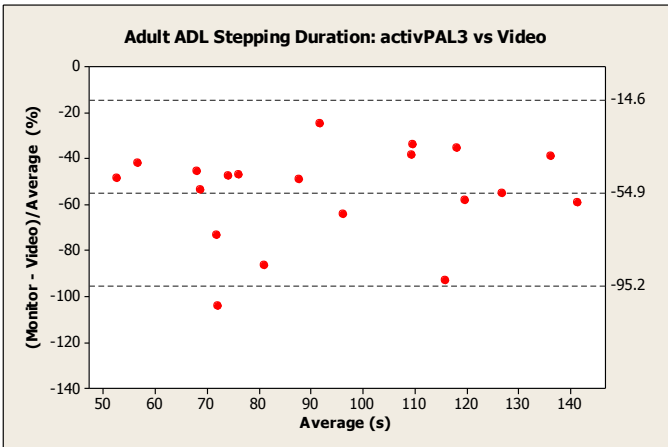

Step count Activities of daily living (ADL)

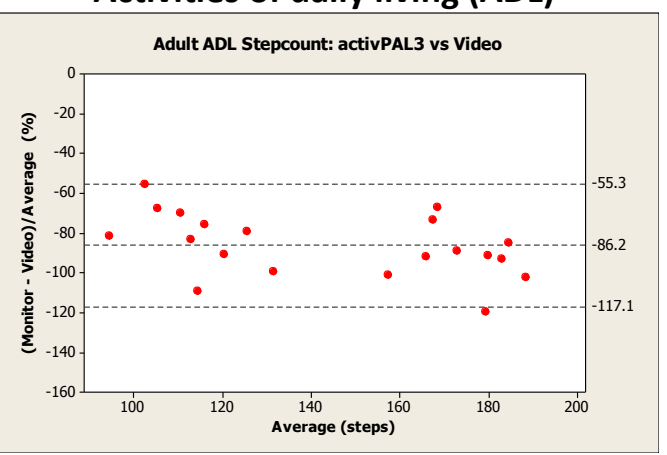


Figure S2 B) Young people - Bland \& Altman Plots (average of monitor and video outcomes against \% difference between outcome

Sitting duration

Standardised activities (STD)

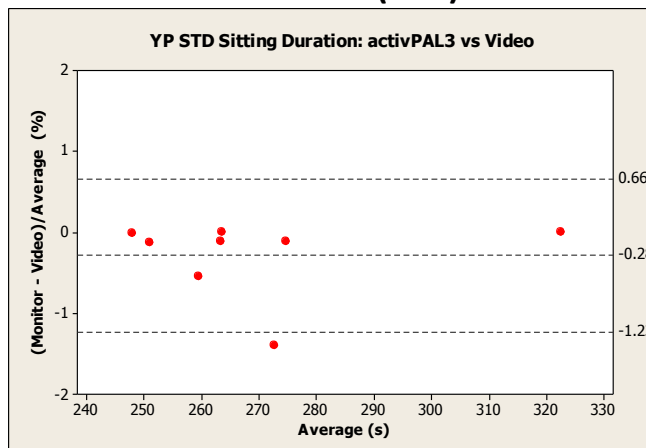

Activities of daily living (ADL)

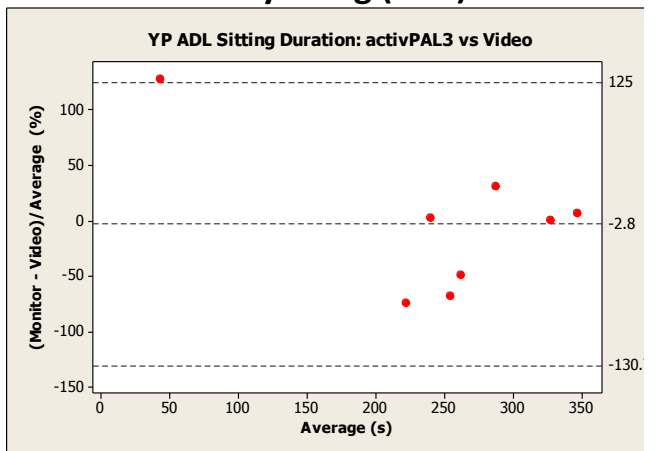

Step count

Standardised activities (STD)

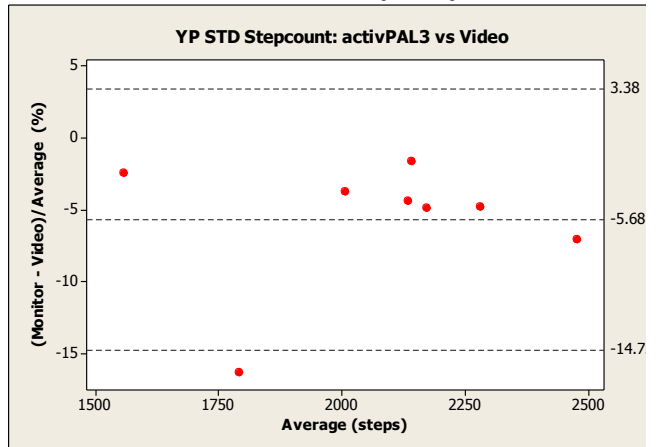

Upright duration
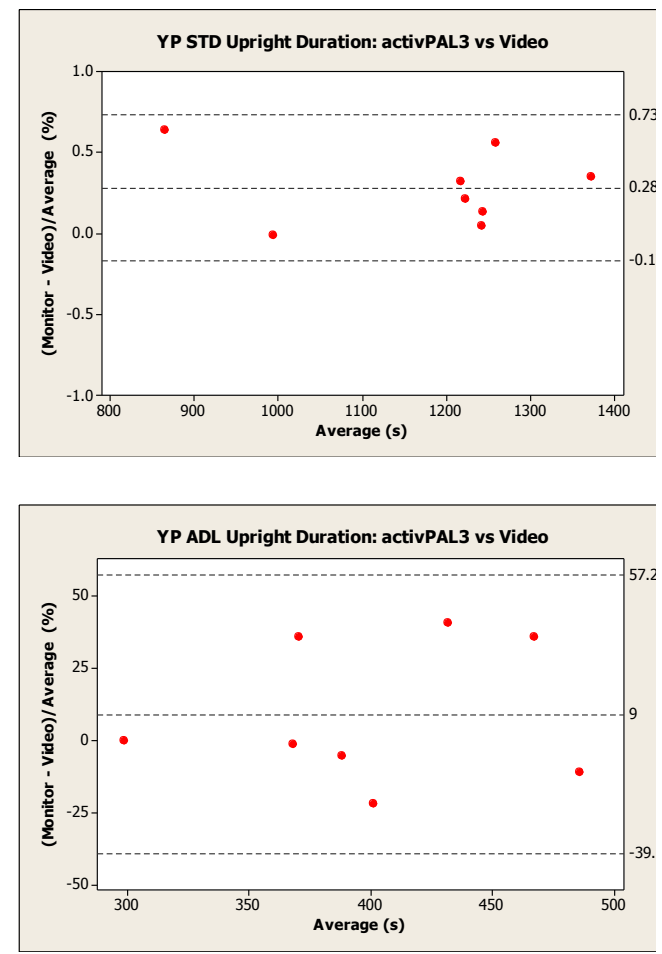

\section{Step count excluding jogging}

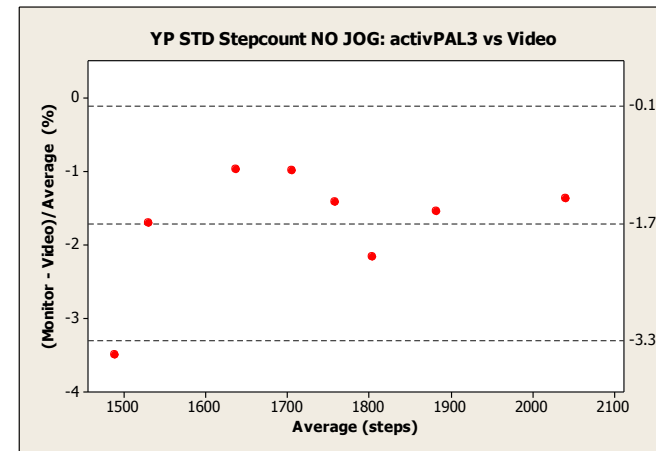

Standing duration
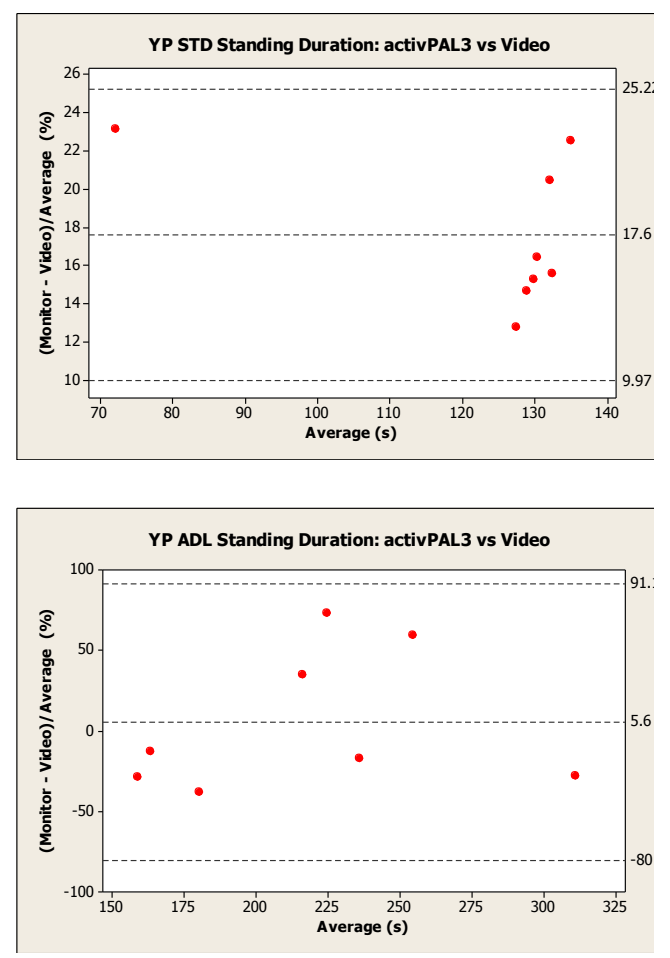

Step count jogging only

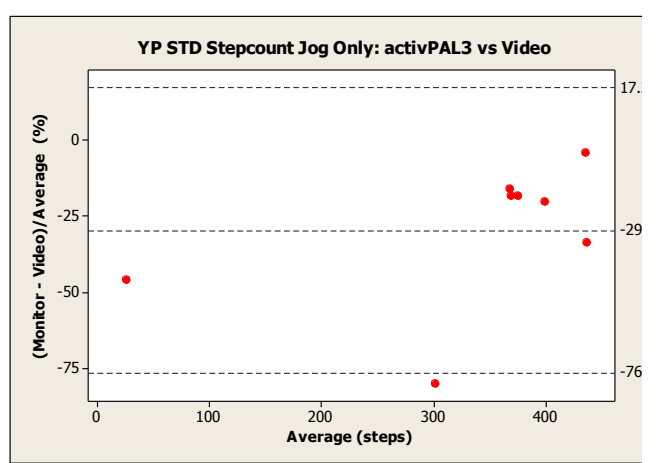

Stepping duration
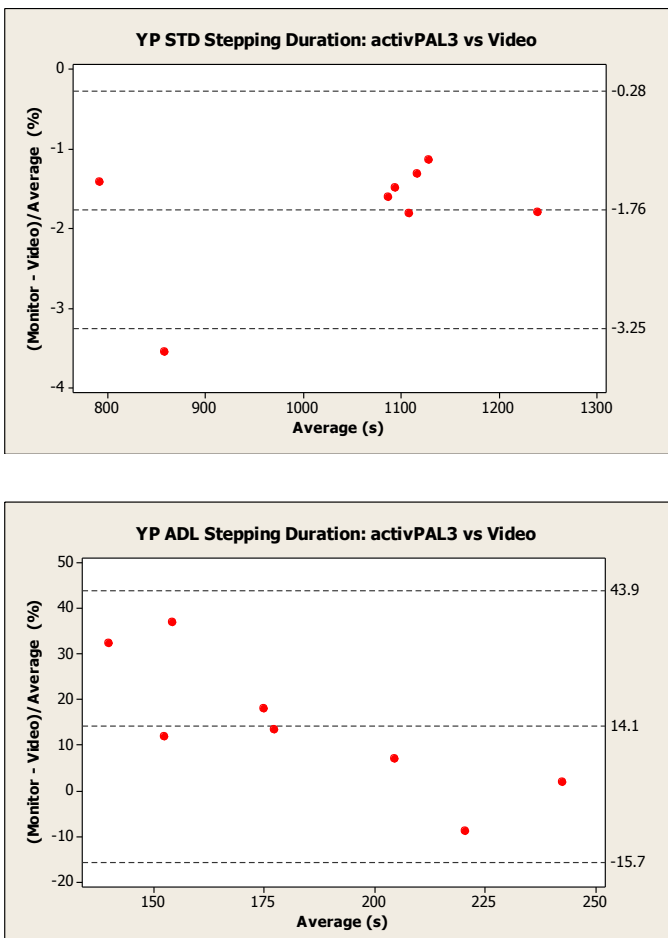

Step count Activities of daily living (ADL)

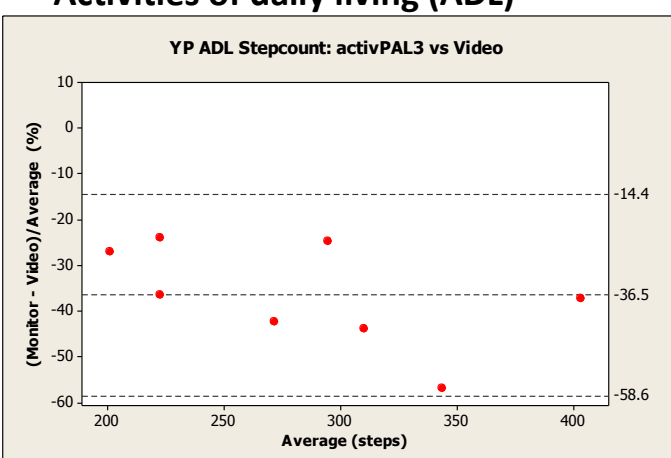




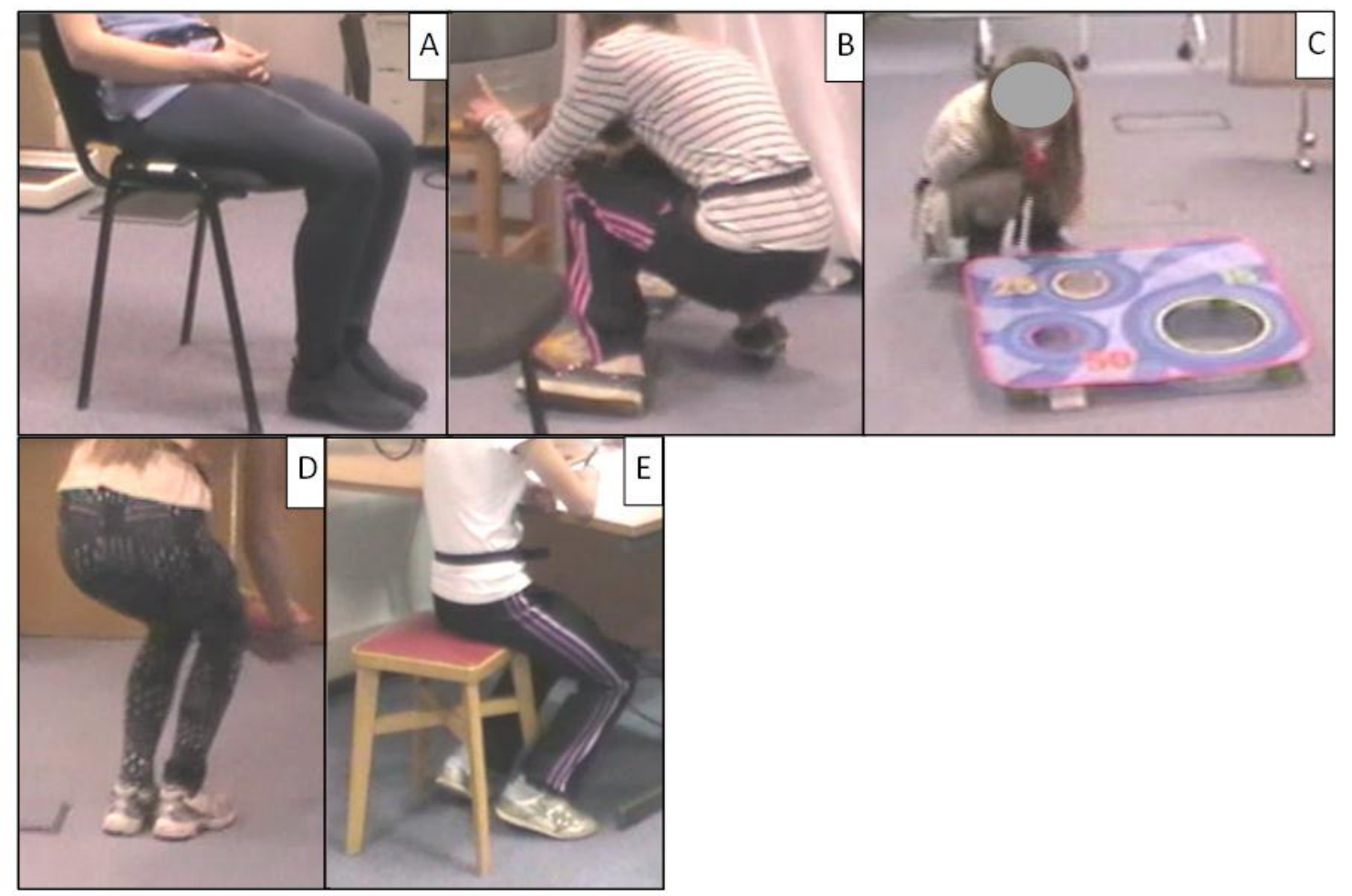

Figure S3: Different classification examples (A adult, B-E young people). A) Video observation=sitting, activPAL3=standing; B-D) video=standing, activPAL3=sitting; E) video=sitting, activPAL3=standing. 


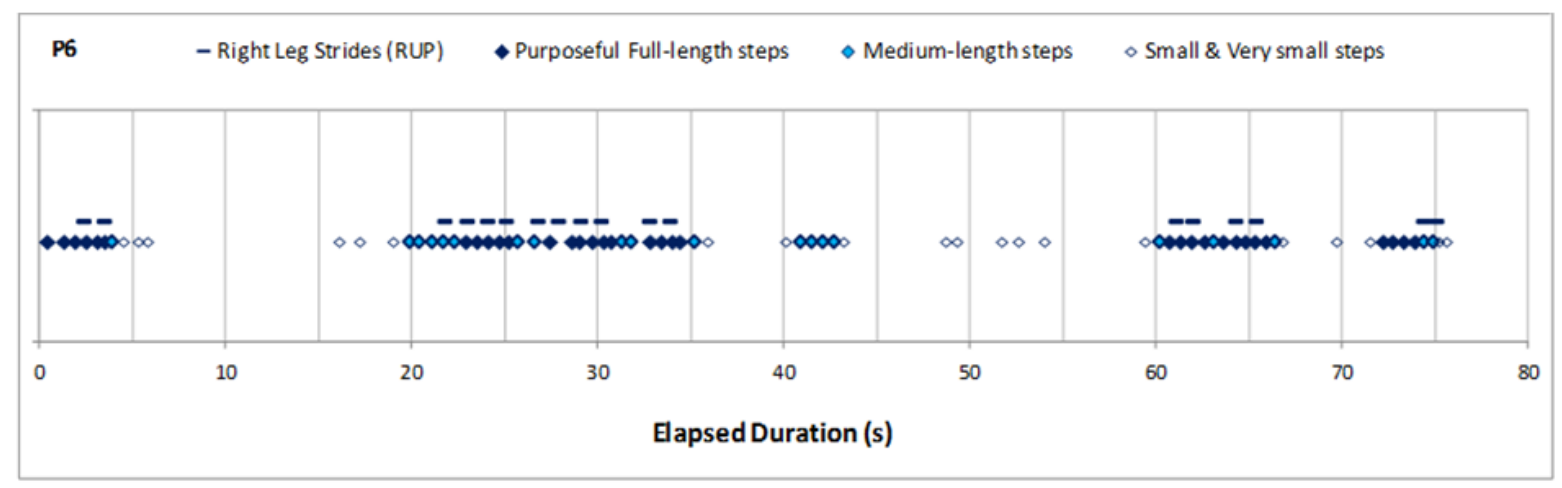

Figure S4: Adult observed steps versus recorded strides for a participant (P6) for the empty rubbish bin ADL activity 


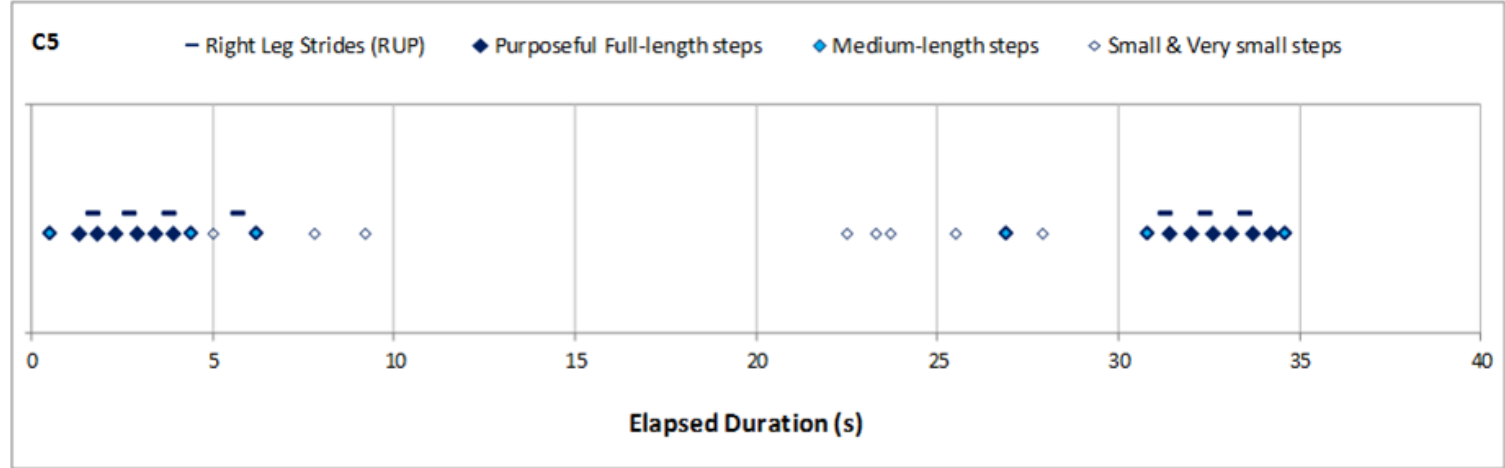

Figure S5: Young people observed steps versus recorded strides for a participant (C5) for the coat On/Off ADL activity 